RePortes de Tesis De Posgrado

\title{
Superficies interactivas flexibles para fomentar el juego cooperativo en niños de preescolar
}

\author{
Vianey Vázquez
}

Publicado: 21 Septiembre 2016

\begin{abstract}
Resumen
Los niños de preescolar tienen dificultades para aprender a trabajar juntos y jugar de forma cooperativa. Las maestras promueven la cooperación para enseñar a los preescolares a completar tareas trabajando en conjunto. Las superficies interactivas son apropiadas para potenciar la colaboración en los niños y usan estímulos multi-sensoriales para mantener su atención. En esta investigación, se pretende identificar los mecanismos de juego cooperativo adecuados para integrarlos en la tela interactiva MúsicaFlexible. Y posteriormente evaluar su capacidad de promover juego cooperativo entre niños de preescolar.
\end{abstract}

Palabras clave: Superficies interactivas; Juego cooperativo; Niños de preescolar; Superficies flexibles.

\section{Introducción}

Los niños de preescolar tienen dificultades para aprender a trabajar juntos. El uso de estrategias de colaboración desde una edad temprana puede enseñar a los preescolares a ser más tolerantes, atentos y respetuosos con los demás y aumentar sus probabilidades de colaborar con otros individuos en su vida cotidiana [6]. En muchos salones de preescolar, las maestras promueven las interacciones y la cooperación para enseñar a los preescolares a guiar y asistir a otros y completar tareas trabajando en conjunto [3].

Las superficies interactivas son apropiadas para el apoyo del desarrollo infantil temprano [8,2]. Las superficies interactivas permiten a los niños interactuar de manera natural con servicios digitales, son apropiadas para potenciar la colaboración y usan estímulos multi-sensoriales para mantener la atención de los niños. En particular, las superficies interactivas flexibles permiten nuevas formas de interacción [5] y pueden apoyar el desarrollo social de los niños [1], sin embargo, no está claro cómo pueden ser útiles para fomentar el juego cooperativo de preescolares y cómo se pueden integrar en los salones de clases de preescolar.

En esta investigación, la hipótesis es que las superficies interactivas flexibles son herramientas apropiadas para apoyar el desarrollo infantil temprano durante actividades de juego

\footnotetext{
Vázquez, V.

Departamento de Ciencias de la Computación

CICESE

Ensenada, B.C. México

Email: esthela@cicese.edu.mx
}

cooperativo en los salones de clases de niños de prescolar.

\section{Trabajo relacionado}

Investigaciones previas sobre superficies interactivas han explorado formas innovadoras para ayudar a los niños a desarrollar habilidades sociales [8], y cognitivas [2] usando mesas y pisos interactivos. Por ejemplo, Stomp [8] es un piso interactivo para apoyar a los niños en el aprendizaje de matemáticas y promover la practicar de actividad física. Un estudio de 2 semanas del uso de Stomp muestra mejoras en activación física, socialización y cognición.

Más allá de la construcción de superficies tradicionales, otros proyectos se han orientado a diseñar nuevas superficies interactivas utilizando materiales deformables como pantallas elásticas, celulares flexibles, y controles suaves [5]. Los investigadores han utilizado tecnología textil inteligente en juguetes, para lograr que niños en edad preescolar los encuentren útiles e intuitivos [7].

Las interfaces deformables permiten nuevas formas de interacción y han sido utilizadas para mejorar la experiencia de usuario, particularmente en experiencias musicales [5], multisensoriales y de socialización. Por ejemplo, Interactive Slide [4] es una rampa de deslizamiento inflable e interactiva que promueve la socialización en niños entre 4 y 6 años. De igual manera, BendableSound [1] es una tela interactiva musical. En un estudio cualitativo con niños de 2 y 3 años mostró que éstos prefieren usarla de manera colectiva. En general, los preescolares perciben las superficies deformables como fáciles de usar, útiles [7], y apropiadas para apoyar sus interacciones [5]. Sin embargo, no se ha explorado la implementación de superficies interactivas flexibles para promover socialización y actividades de juego cooperativo en las aulas de niños de preescolar.

\section{Objetivo de investigación}

Es importante que los niños desarrollen sus habilidades sociales y cognitivas, y realizar tareas en conjunto puede contribuir a ello [4], por lo que el objetivo de esta investigación es: Diseñar e implementar mecanismos de juego cooperativo apropiados para la tela interactiva MúsicaFlexible, y evaluar su capacidad de promover juego cooperativo entre preescolares.

De acuerdo al objetivo mencionado, se tienen las siguientes preguntas de investigación:

- ¿Cuáles son las actividades y mecanismos para juego cooperativo más adecuadas para implementarse en una superficie interactiva flexible que promueva directamente el juego cooperativo entre preescolares? 
- ¿Cuál es la experiencia de uso y utilidad de las superficies interactivas flexibles para promover el juego cooperativo en preescolares dentro del salón de clases?

- ¿Cómo mejora la práctica de juego cooperativo entre preescolares la integración de mecanismos para juego cooperativo en MúsicaFlexible?

\section{Metodología}

Para responder las preguntas de investigación propuestas, estoy siguiendo una metodología centrada en el usuario usando un enfoque de métodos mixtos, que incluye métodos cualitativos y cuantitativos.

En primer lugar, realizaré un estudio contextual para identificar los problemas que los preescolares enfrentan durante el juego paralelo y colaborativo dentro de aulas de clases; y entender las estrategias de los preescolares y sus maestras para resolver estos problemas. Actualmente me encuentro en la etapa de recolección de información y entrevistas para el estudio contextual. Como resultados de este estudio se esperan un conjunto de escenarios que muestren la utilidad de una superficie interactiva flexible en el apoyo a la práctica del juego cooperativo en niños de preescolar.

Después, usaré esos escenarios para diseñar de manera iterativa, actividades que sean adecuadas para promover el juego cooperativo. Realizaré múltiples sesiones de diseño participativas para definir prototipos de baja fidelidad, los cuales serán evaluados con los usuarios potenciales. Con base en los resultados de la evaluación, voy a desarrollar un conjunto de mecanismos de colaboración que se puedan integrar a una superficie flexible e implementaré el prototipo para realizar una prueba de campo.

Finalmente, evaluaré la usabilidad y utilidad de la superficie interactiva flexible en un estudio en sitio. El prototipo se evaluará en un jardín de niños para demostrar cómo la superficie flexible promueve la práctica de juego cooperativo y apoya el desarrollo social de los preescolares.

\section{Resultados actuales}

En los últimos meses hemos seguido una metodología centrada en el usuario para diseñar e implementar el prototipo MusicaFlexible [1], una tela interactiva que permite a los niños reproducir sonidos de piano cuando golpean y tocan la tela, o cuando mueven los elementos digitales que aparecen sobre ella. Las animaciones de MúsicaFlexible muestran un fondo 3D con la animación de una nébula y elementos traslúcidos del espacio, como naves espaciales y estrellas, desplegadas en una tela elástica que actúa como pantalla digital [1].

Actualmente estoy iniciando el estudio contextual, principalmente la etapa de colección de datos. He realizado 3 entrevistas semi-estructuradas a maestras de preescolar, con el objetivo de entender cómo es la socialización de los niños en edad preescolar en el aula y cuáles estrategias utilizan los maestros para promoverla.

Estas entrevistas se analizaron y codificaron mediante tres tipos de codificación (i.e., abierta, axial y selectiva). La categorización de la información de las entrevistas será analizada con grupos focales tomando en cuenta los resultados obtenidos en un estudio de despliegue de MusicaFlexible [1], durante observación participativa (10 horas) y 5 grupos focales con maestros de preescolar (2.5 horas).

\section{Resultados esperados}

Como trabajo a futuro llevaré a cabo todas las etapas planteadas en la metodología. Terminaré de realizar el estudio contextual, para proponer alternativas de diseño para la tela interactiva. Después, desarrollaré un prototipo robusto para poder implementarlo y por ultimo evaluar su utilidad y el valor de este prototipo para el salón de clases de preescolar. En cuanto a posibles contribuciones, se espera proveer evidencia empírica de que la tela interactiva flexible que se diseñe pueda apoyar la socialización, promover la práctica de juego cooperativo y potenciar el desarrollo social de niños en edad preescolar.

\section{7 ¿Con qué necesito ayuda?}

Este consorcio de posgrado es muy importante para mi investigación porque en este momento me encuentro en la primera etapa de la realización de mi tesis de maestría. Considero que es una buena oportunidad para poder recibir sugerencias y guías expertos en IHC. Me gustaría recibir retroalimentación sobre la significancia de mi trabajo y si los métodos seleccionados son apropiados. Esta retroalimentación me puede ayudar a obtener un mejor resultado y que se muestre en la tesis.

Para seguir avanzando en mi investigación de la mejor manera posible, me gustaría obtener retroalimentación en términos de:

- Metodologías de diseño para niños o, incluso, usuarios no verbales o con dificultades de comunicación, ya que al tratarse de niños pequeños es probable que no logren comunicar sus ideas y preferencias de manera explícita durante las sesiones de diseño participativas.

- Principios de diseño para lograr engagement en los niños.

- Herramientas y/o técnicas para la medición y evaluación de avances o cambios en el desarrollo de socialización en niños.

\section{Referencias}

[1] Cibrian, F. L., Weibel, N., \& Tentori, M. (2016, September). Collective use of a fabric-based interactive surface to support early development in toddler classrooms. UBICOMP 2016 (pp. 328-339). ACM.

[2] Piper, A.M. et al. 2012. TAP \& PLAY: An End-User Toolkit for Authoring Interactive Pen and Paper Language Activities. CHI '12, 149.

[3] Slavin, R.E. 1987. Developmental and Motivational Perspectives on Cooperative Learning: A Reconciliation. Child Development. 58, 5 (1987), 1161-1167.

[4] Soler-Adillon, J. et al. 2009. A novel approach to interactive playgrounds: the interactive slide project. IDC '09 , 131-139.

[5] Troiano, G.M. et al. 2015. Deformable Interfaces for Performing Music. Proceedings of the 33rd Annual ACM Conference on Human Factors in Computing Systems (New York, NY, USA, 2015), 377-386.

[6] UNICEF 2004. Desarrollo Psicosocial de los niños y las niñas. Colombia: UNICEF.

[7] Vega-Barbas, M. et al. 2015. Utilizing Smart TextilesEnabled Sensorized Toy and Playful Interactions for Assessment of Psychomotor Development on Children. Journal of Sensors. 2015, (2015), 1-9.

[8] Wyeth, P. et al. 2013. Activity, motivation and games for young children. Proceedings of The 9th Australasian Conference on Interactive Entertainment Matters of Life and Death - IE '13 (2013), 1-3. 